\title{
Methodology for the Experimental Determination of the Powertrain's Inertia Ellipsoid and its Verification
}

\author{
Pavel Brabec ${ }^{1, a}$ \\ ${ }^{1}$ Technical University of Liberec, Dept. of Vehicles and Engines, Studentská 2, 46117 Liberec 1, Czech Rep.
}

\begin{abstract}
This paper summarizes current scientific knowledge and the results of a study focused on the determination of a powertrain's inertia ellipsoid. The work deals with the application of methods for the experimental determination of the inertia matrix and summarizes their basic potential. The work describes a proposed computational algorithm by means of which the inertia ellipsoid can be determined. The experimental section of the work presents the results of measurements for internal combustion engines (powertrains).
\end{abstract}

\section{Introduction}

A number of methods have been used to determine the moments of inertia. All of the methods are based on a principle of relationship between the body's moment of inertia and the frequency of the oscillation. The measurement process assumes the oscillation to be undamped and the time of the oscillation is measured. The fundamental methods for determining the body's moment of inertia are based on the principle of the compound pendulum, torsional suspension unit or bifilar suspension apparatus (and/or trifilar or quadrifilar suspension apparatus, as the case may be).

The work was aimed at propose a methodology for measuring on the basis of which the main mass parameters of the internal combustion engine and transmission system (powertrain) could be identified. The measured powertrain's moment of inertia matrices may be further used in practice as one of the input parameters for the simulation and computation. These data can be primarily used for optimizing the powertrain's flexible mount assembly and simulating the vehicle's crash tests.

An effective solution to the above mentioned problem can be the application of accurate input parameters to the simulation and computation both from the view point of safety (crashtests) and comfort (quality powertrain mount assembly in the car body).

Therefore an experimental stand for determining the mass, center-of-gravity position and moments of a powertrain's inertia was created where necessary measurements (with the greatest degree of possible accuracy) were later carried out.
Figure 1. The powertrain's mount assembly and vehicle's crashtest simulation results $\operatorname{crash}^{[7]}$.
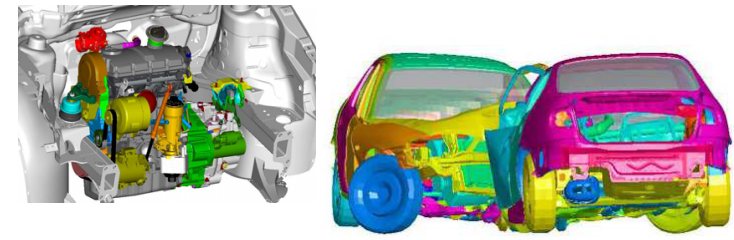

\section{The Determination of the Moments of Inertia}

The moments of inertia and the deviation moments of inertia can be expressed in a matrix form using one quantity - the inertia matrix,

$\mathbf{I}=\left[\begin{array}{ccc}I_{x} & -D_{x y} & -D_{x z} \\ -D_{y x} & I_{y} & -D_{y z} \\ -D_{z x} & -D_{z y} & I_{z}\end{array}\right]$,

which is the matrix of the second-order symmetrical tensor.

\subsection{The Inertia Ellipsoid and Principal Axes of Inertia [2][5]}

As the axis passing through the selected origin changes its direction, the body's moment of inertia will change.

\footnotetext{
${ }^{a}$ Corresponding author: pavel.brabec@tul.cz
} 
Let us plot the reverse square roots of the relevant moments of inertia on these axes from the origin $O M=\sqrt{\frac{1}{I_{o}}}$ and search for the geometrical locus of end points $\mathrm{M}$. By means of the coordinates ( $\mathrm{x}, \mathrm{y}, \mathrm{z})$ of a point denoted as $M$, the relationship for the moment of inertia with respect to any axis passing through the origin of the Carthesian coordinate system can be modified to

$I_{x} \cdot x^{2}+I_{y} \cdot y^{2}+I_{z} \cdot z^{2}-2 \cdot D_{x y} \cdot x \cdot y-2 \cdot D_{y z} \cdot y \cdot z$ $-2 \cdot D_{z x} \cdot z \cdot x=1$.

The locus sought after is the ellipsoid (generally the triaxial ellipsoid). It is called the inertia ellipsoid (hereinafter referred to as ES).

Figure 2. The Inertia Ellipsoid.

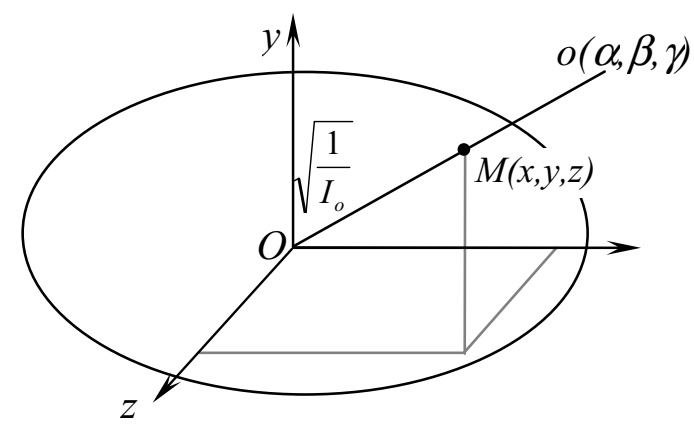

The axes of the ellipsoid are called the principal axes of inertia and then, a condition applies that all three deviation moments with respect to these axes are equal to zero. The moments with respect to these axes are called the principal moments of inertia.

\subsection{Experimental Determination of the Moments of Inertia}

The moments of inertia can be determined by means of any CAD software if a 3-D model of the component exists. The relevant software requires the bodies'material density values and the appropriate command will compute the centre-of-gravity position and inertia matrices. However, we may face a situation when this approach cannot be used and consequently, the moments of inertia will have to be determined experimentally.

\section{Description of Method}

Measurement accuracy represented a key aspect for selecting a method for determining the powertrain's inertia ellipsoid. The correctly determined powertrain's inertia matrix is critical for further use in computer simulations, e.g. crashtests.

The determination of the mass and the center of gravity of the powertrain and/or the engine was carried out on the basis of the measurement on a trifilar suspension apparatus by measuring tensile forces in individual wires. To determine the moments of inertia, the indirect method which measures the period of oscillation of the body suspended from a unifilar (torsional) suspension apparatus was used.

To determine the moments of inertia for the bodies having more complex shapes we will definitely need fixtures that will enable suspension of the body in various positions from the torsional suspension apparatus. Therefore, an auxilliary frame for clamping the powertrain (engine) was made. The powertrain was clamped into the auxilliary frame in a defined way so that the axes of the selected powertrain's coordinate system were parallel to the axes of the frame's coordinate system.

Since the frame's moments of inertia are not insignificant, two measurements were needed. The first measurement was made for the frame-powertrain system. The other measurement was carried out separately for the frame so that the frame could be ,subtracted" from the measured system. To determine the moments of inertia, the indirect method measuring the period of oscillation of the body suspended from a unifilar (torsional) suspension apparatus was used. The formulae for the computation of the moment of inertia using a torsional suspension apparatus is as follows:

$I=\frac{G \cdot d^{4}}{128 \cdot \pi \cdot L} \cdot T^{2}$

Where $I$ - the moment of inertia, $G$ - spring wire's modulus of elasticity in shear, $d$ - spring wire diameter, $L$ - suspension length, $T$ - time period of one oscillation.

The measurements necessitated development of a suspension apparatus which consisted of universal joints (Cardan joints) and a spring wire. The ends of the suspension apparatus were equipped with carrying yokes into which the spring wire was fixed by clamping. The structure enables the measured body to hang freely (the line passing through the wire axis intersects the body's centre of gravity) for the whole period of the oscillation and for all cases of the suspension (See Fig.3).

The measured quantities were used for the determination of elements of inertia matrix of the powertrain and auxilliary frame. At least six measurements are needed to determine the inertia matrix. However, at least ten measurements were taken to increase the result accuracy based on the relationship determining the moment of inertia with respect to any axis passing through the origin. Consequently, the position of the inertia ellipsoid was determined by means of other mathematical procedures.

If we plot the values of $\sqrt{\frac{1}{J}}$ from the origin using a specific scale as vectors along the axis of the suspension apparatus, the end points will form an 
ellipsoid of inertia in a space. The following simplified picture shows a process for plotting the inertia ellipsoid in the plane passing through the axis of the crankshaft and axes of the cylinders. Naturally, to enhance the accuracy, more measurements should be taken, ideally a few measurements using the suspension apparatuses which determine the points of the inertia ellipsoid in the proximity of ends of its half-axes (i.e. around the vertices of ellipse).

Figure 3. Principle of plotting an inertia ellipsoid in a plane of the crankshaft.

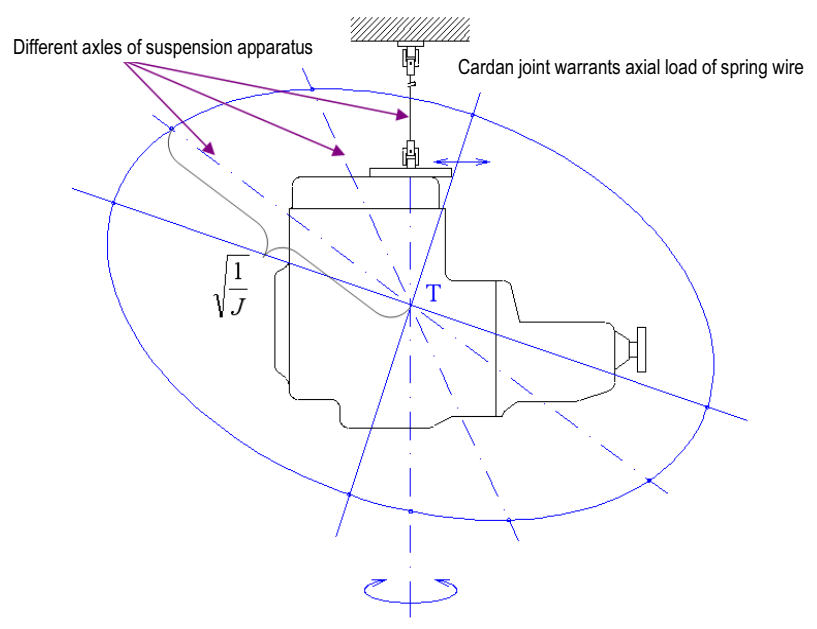

Let us point out that to determine the inertia matrix (i.e. the inertia ellipsoid as well) we have to oscillate the body around at least six different axes using different suspension points. The directions of the individual axes should differ from each other as much as possible. Consequently, we would get six values of the moments of inertia with respect to different axes passing through the centre of gravity and we could determine the inertia matrix of the measured body by means of the a system of equations. Six equations involving six unknowns (using equation (2)) suffice for computing the moments of inertia with respect to the $\mathrm{X}, \mathrm{Y}$ and $\mathrm{Z}$ axes and deviation moments.

For measurement accuracy reasons and for the follow-up computation of the inertia ellipsoid's size and position (hereinafter ZVES - i.e. fundamental properties of the inertia ellipsoid, i.e. the principal moments of inertia and the position of inertia ellipsoid's axes) at least ten measurement of the moment of inertia were taken with respect to any axis passing through the origin. In general, the matrix of n-equations in six unknowns will be obtained for n-measurements. In this event, the unknowns $\left(I_{X}, I_{Y}, I_{Z}, D_{X Y}, D_{Y Z}\right.$ and $D_{Z X}$ ) can be determined by the method of least squares ${ }^{[8],[9]}$.

The method of least squares is a mathematical method for statistical data processing. It helps find a suitable approximation function for given, empirically determined values. The function being searched for must be a linear combination of the functions which were known in advance and the method will enable their coefficients to be computed. The method of least squares seeks out such values of the coefficients so that the sum of the squares of their functional value deviations from the given measured values was as low as possible. In a more simplified way: „so that the sum of the squares of the deviations was the lowest". We can make up a system of quotations $\mathbf{A} \cdot \mathbf{x}=\mathbf{b}$ ( $\mathbf{A}$ is a matrix which we know, $\mathbf{b}$ is a vector which we know, too, $\mathbf{x}$ is an unknown vector).

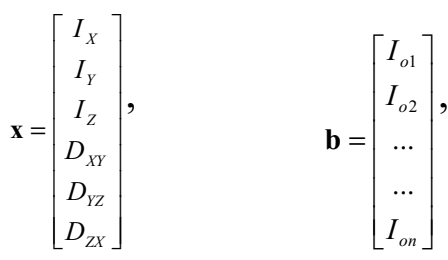

$\mathbf{A}=\left[\begin{array}{cccccc}\cos ^{2} \alpha_{1} & \cos ^{2} \beta_{1} & \cos ^{2} \gamma_{1} & -2 \cdot \cos \alpha_{1} \cdot \cos \beta_{1} & -2 \cdot \cos \beta_{1} \cdot \cos \gamma_{1} & -2 \cdot \cos \gamma_{1} \cdot \cos \alpha_{1} \\ \cos ^{2} \alpha_{2} & \cos ^{2} \beta_{2} & \cos ^{2} \gamma_{2} & -2 \cdot \cos \alpha_{2} \cdot \cos \beta_{2} & -2 \cdot \cos \beta_{2} \cdot \cos \gamma_{2} & -2 \cdot \cos \gamma_{2} \cdot \cos \alpha_{2} \\ \ldots & \ldots & \ldots & \ldots & \ldots & \ldots \\ \ldots & \ldots & \ldots & \ldots & \ldots & \ldots \\ \cos ^{2} \alpha_{n} & \cos ^{2} \beta_{n} & \cos ^{2} \gamma_{n} & -2 \cdot \cos \alpha_{n} \cdot \cos \beta_{n} & -2 \cdot \cos \beta_{n} \cdot \cos \gamma_{n} & -2 \cdot \cos \gamma_{n} \cdot \cos \alpha_{n}\end{array}\right]$

In the present case the matrix $\mathbf{A}$ has more rows than columns, the size of the vector $\mathbf{b}$ is bigger than the one of the vector $\mathbf{X}$. It is an over determined system of equations which has more equations than unknowns. In this event it is theoretically possible to ignore some equations but let us suppose that the equations result from experimental data where each measurement is considered valuable and we are not able to decide which of these laboriously obtained equations should be ignored.

In the cases of the over determined system of linear equations such a system has an infinite number of solutions (or more precisely, no solution). Let us say that such a fact is not satisfactory and define an error in solution $\mathbf{e}=\mathbf{A} \cdot \mathbf{x}-\mathbf{b}$.

We want to find such a solution $\mathbf{x}$, so that the error (or more precisely the size of the vector e ) was minimal. The solution of our system of equations can be derived by derivation of matrices and vectors. Since $\mathbf{e}$ is a vector, let us adapt the requirement so that the sum of the squares of the individual deviations (i.e. the elements of the vector $\mathbf{e}$ ) is „minimal“. Defining a criterium in such a way actually results in the minimization of the scalar product which can be written by means of transposition.

$$
\mathbf{e}^{T} \cdot \mathbf{e} \rightarrow \min
$$

The product will be minimal if its derivation according the variable will equal zero.

$$
\left(\mathbf{e}^{T} \cdot \mathbf{e}\right)^{\prime}=\left[(\mathbf{A} \cdot \mathbf{x}-\mathbf{b})^{T} \cdot(\mathbf{A} \cdot \mathbf{x}-\mathbf{b})\right]^{\prime}=0 \text {. The relationship }
$$
can be further modified using the rules for transposition of products and derivation of the products of matrices and vectors.

$\left[\mathbf{x}^{T} \cdot \mathbf{A}^{T} \cdot \mathbf{A} \cdot \mathbf{x}-\mathbf{x}^{T} \cdot \mathbf{A}^{T} \cdot \mathbf{b}-\mathbf{b}^{T} \cdot \mathbf{A} \cdot \mathbf{x}+\mathbf{b}^{T} \cdot \mathbf{b}\right]^{\prime}=2 \cdot \mathbf{A}^{T} \cdot \mathbf{A} \cdot \mathbf{x}-2 \cdot \mathbf{A}^{T} \cdot \mathbf{b}=0$ 
The relationship above may be used to express the resultant formulae for $\mathbf{x}$.

$$
\mathbf{x}=\left(\mathbf{A}^{T} \cdot \mathbf{A}\right)^{-1} \cdot \mathbf{A}^{T} \cdot \mathbf{b}
$$

Determination of the centre-of-gravity position was made by means of measurement on a trifilar suspension apparatus (measuring the tensile forces in individual wires). Firstly, the centre-of-gravity position was determined in one plane XY, then the frame (or the powertrain-frame assembly) turned and the centre-ofgravity position was determined in the plane perpendicular to the original plane (YZ).

The measurement described above i.e. the determination of the centre-of-gravity position from the origin along all three axes will be carried out separately for the frame as well as for the assembly (the frame and the powertrain). Consequently, the measured and computed values can be used to compute the mass and the position of the powertrain's centre-of-gravity.

The last computational operation which has to be performed to determine the inertia matrix of the powertrain (engine) is subtraction of the auxilliary frame's inertia matrix from the frame-powertrain assembly's inertia matrix. Steiner's theorem will be used for this computation.

Figure 4. Illustration of the measured inertia ellipsoid.
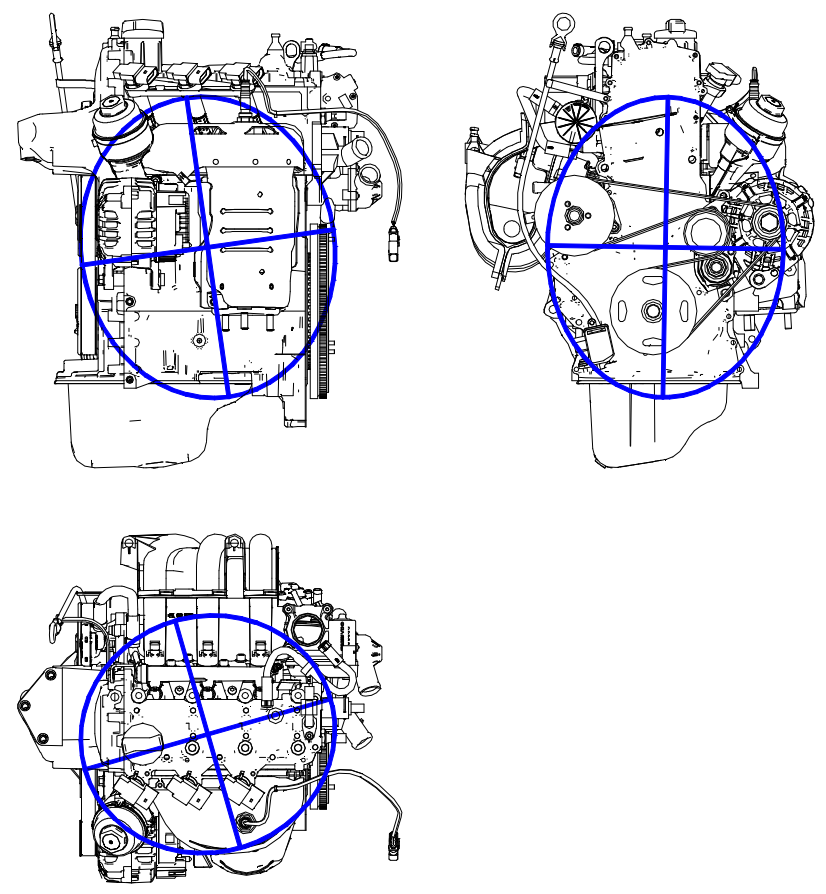

4 Measurement Accuracy

\subsection{Measurement verification using a simple body clamped in the frame}

To verify the measurement method the following procedure was selected. Firstly, a geometrically simple body was chosen and the body passed through the complete measurement process as a separate powertrain i.e. firstly, the frame-body assembly and then the frame itself were measured. A simple form of the body was selected due to a simple calculation of the body's moment of inertia matrix which was further compared with the measured matrix. Considering the semi-finished products being available in our laboratory, an assembly consisting of two semi-finished products $(\varnothing 100$ and $\varnothing 130 \mathrm{~mm}$ ) was chosen. The position of the semi-finished products was chosen in such a way so that one of the deviation moments was not zero. See the Figure below.

Figure 5. Semi-finished products for the measurement verification.

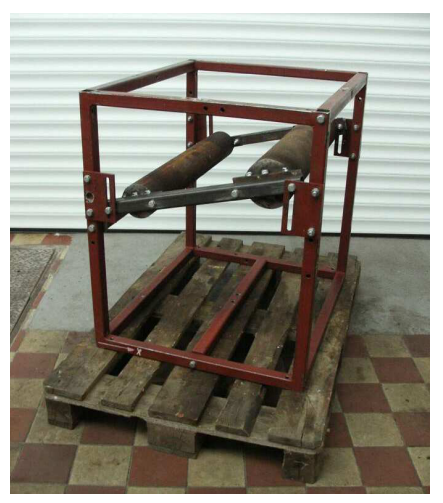

The following evaluation of the results

$\Delta=\frac{H_{N}-H_{V}}{H_{V}} \cdot 100[\%]$

$\left(H_{N}-\right.$ measured value; $H_{V}-$ computed value using $3 \mathrm{D}$ CAD), will result in the following relative errors in measurements

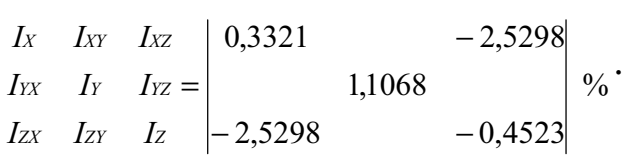

The principal moments of inertia showed very good conformity, the most significant deviation occurred along the $\mathrm{x}$-axis and it was less than $\mathbf{1 . 5} \%$.

The position of the semi-finished products can be considered asymmetrical. However, the asymmetry is chosen in such a way that two deviation moments are zero. For these two deviation moments the relative error cannot be determined, we are able to determine only the absolute one (i.e. $-0,0895 \mathrm{~kg} \cdot \mathrm{m}^{2}$ and $-0,0198 \mathrm{~kg} \cdot \mathrm{m}^{2}$ ). The last deviation moment was measured approximately with the relevant error of $\mathbf{2 . 5} \%$. This result is very good taking into account the comparison of the deviation moments'sizes (comparing with the principal moments of inertia, lower orders of values are in question). 


\subsection{Experimental determination of the position of the principal axis of inertia for a semi-finished product situated ,across" the frame}

By further measurement to verify the method, a semifinished product having the dimensions of Ø60-1500 mm was located into the position of the auxilliary frame diagonal. The measurement method matched the verification described in the previous section (i.e. the method which included the powertrain as well). Firstly, the moments of inertia matrix of the chosen semi-finished product was computed using the CAD software and then it was determined by means of measurements. These moments of inertia matrices served for further determination and comparison of the solid angles of the principal axis of inertia from the coordinate system. See the following illustration.

Figure 6. Photograph and CAD model with a semifinished product located diagonally.
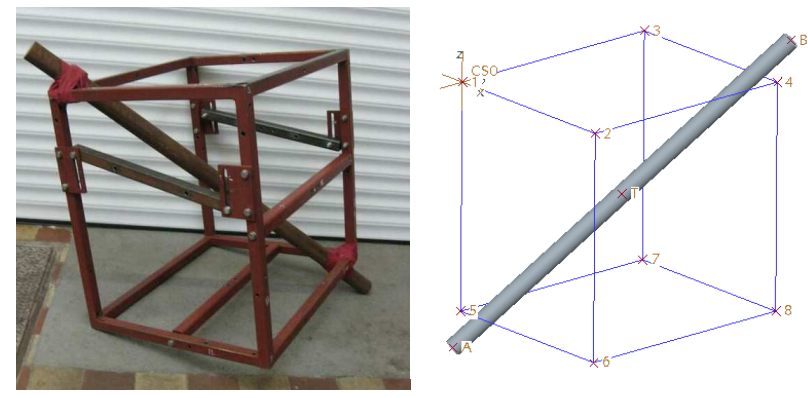

Using the same evaluation of the results as for previous cases (see formula 5), we can write: the following relative errors of measurement:

$$
\begin{aligned}
& \begin{array}{llllll}
I_{X} & I_{X Y} & I_{X Z} & -0,2455 & 0,6231 & -0,6673
\end{array}
\end{aligned}
$$

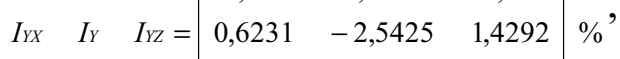

$$
\begin{aligned}
& \begin{array}{lll|lll}
I_{Z X} & I_{Z Y} & I_{Z} & -0,6673 & 1,4292 & 1,1100
\end{array}
\end{aligned}
$$

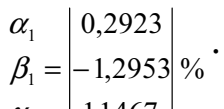

$$
\begin{aligned}
& \gamma_{1}|1,1467|
\end{aligned}
$$

A comparison of the computed and measured moments of inertia values resulted in an average error of $1.10 \%$, the max. deviation occurred along the y-axis $(2.54 \%)$. The absolute error along the principal axis of inertia (identical with the semi-finished product axis) was less than $1^{\circ}$ for all solid angles.

\subsection{The influence of the air filter on the inertia ellipsoid of powertrain for passenger car}

Further measurements were carried out two variants of the powertrain, which consisted of $1.459 \mathrm{~kW}$ MPI engine and gearbox MQ 200. First unit was measured without the air filter and later was measured second variant with intake filter. Weight of the plastic intake filter was $1.17 \mathrm{~kg}$ (which is approximately $0.9 \%$ of the weight of the aggregate).

Even with such a small change in weight (but at a great distance from the center of gravity) occurred in all ten measuring of the oscillation period of the assembly (i.e. frame + powertrain) in a different position to a measurement a long time for time period of one oscillation of powertrain with intake filter. Differences measured times one oscillation were around $0.6 \%$. Measured values are shown in the following table.

Table 1. Spatial angles and times of one oscillation.

\begin{tabular}{|c|c|c|c|c|c|}
\hline$\alpha\left(^{\circ}\right)$ & $\beta\left(^{\circ}\right)$ & $\gamma\left(^{\circ}\right)$ & $\begin{array}{c}\text { without } \\
\text { filter } \\
\mathrm{T}_{\mathrm{BF}}(\mathrm{s})\end{array}$ & $\begin{array}{c}\text { with } \\
\text { filter } \\
\mathrm{T}_{\mathrm{SF}}(\mathrm{s})\end{array}$ & $\begin{array}{c}{\left[\left(\mathrm{T}_{\mathrm{BF}}-\mathrm{T}_{\mathrm{SF}}\right) /\right.} \\
\left.\mathrm{T}_{\mathrm{BF}}\right] .100\end{array}$ \\
\hline 62,17 & 53,58 & 131,17 & 10,822 & 10,89 & 0,63 \\
\hline 122,75 & 55,75 & 128,08 & 10,588 & 10,626 & 0,36 \\
\hline 63,42 & 130,42 & 127,83 & 9,373 & 9,449 & 0,81 \\
\hline 120,83 & 128,25 & 126,67 & 9,969 & 9,988 & 0,19 \\
\hline 122,67 & 130,33 & 57,67 & 10,76 & 10,835 & 0,70 \\
\hline 93,5 & 49,17 & 138,92 & 10,529 & 10,583 & 0,51 \\
\hline 54,17 & 142,33 & 80,08 & 9,753 & 9,83 & 0,79 \\
\hline 92 & 13,25 & 104,17 & 9,374 & 9,447 & 0,78 \\
\hline 5,42 & 94,08 & 93,83 & 11,217 & 11,267 & 0,45 \\
\hline 94,08 & 94,42 & 6,08 & 10,69 & 10,725 & 0,33 \\
\hline
\end{tabular}

If these changes in inertia matrix are expressed as a percentage according to the formula $\left[\left(I_{S F}-I_{B F}\right) / I_{B F}\right] \cdot 100$, so we come out

$$
\left|\begin{array}{ccc}
1,33 & 1,52 & -49,79 \\
1,52 & 3,84 & 1,35 \\
-49,79 & 1,35 & 0,65
\end{array}\right|[\%] .
$$

\section{Conclusions}

The presented work deals with an experimental determination of the body's moment of inertia. The work focuses on evaluating the data obtained from measurements carried out on various types of powertrains or engines. To meet the objectives, a measurement method to determine the moments of inertia, centre-ofgravity position and the powertrain mass was developed. Then specialized aids for measurements were developed together with a special program for evaluating the measured data.

The method was verified by measuring several powertrains of engines, in some cases for more versions of the engine accessory arrangement (twelve powertrains and one engine used for passenger cars, one engine used for a commercial vehicle). The measurement was always performed without media i.e. without oil and coolant.

The metering of inertia moment is well known, but measurement accuracy represented a key requirement in 
the measurement method design. Consequently, more values were always measured and for the determination of the ellipsoid of inertia an approximation using the method of least squares was used.

The mathematical derivation which considered all adverse impacts to a maximum extent resulted in approximately $6 \%$ measurement accuracy. A simpleshaped body was used for the complete measurement process calibration. The relative error between the measured values and the CAD program computed values was up to $2.5 \%$.

The mass (in the form of engine's accessories) being added to or removed from the engine affects the resulting values of the moments of inertia and positions of the centres of gravity. The change in the moment of inertia depends on the following factors: the moment of inertia of the relevant component, the mass $(m)$ and the distance from the centre of gravity $(e)$. The relation of $I_{0}=I_{0 S}+m \cdot e^{2}$ is known as the Steiner formula ${ }^{[2]}$. The distance from the centre of gravity plays a significant role because even a relatively small mass can affect the total moment of inertia of the power train. Several versions of measurements of engines and powertrains with varied arrangement were chosen to assess the impact of accessories. E.g. the measurement carried out on the truck engine (3.8 1 Avalon Engine) resulted in nearly $20 \%$ change in the axial moment of inertia when approximately a $10 \%$ change in mass occurred.

Figure 7. Comparison of the inertia ellipsoid of the engine with and without a compressor for air conditioning.

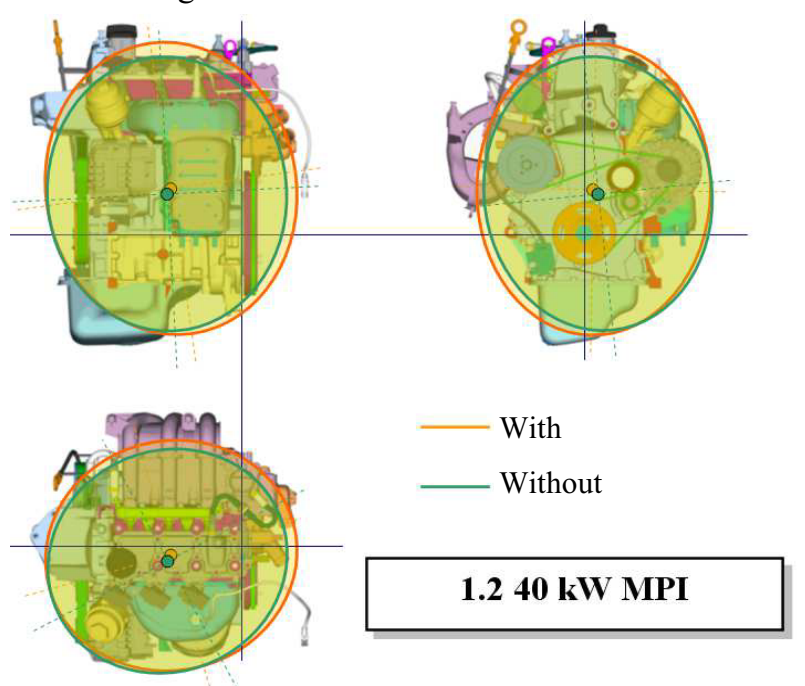

The proposed measurement methodology was able to reveal even a relatively small difference in measurement depending on the powertrain's accessories. Consequently, the change resulting from removal of the engine's intake filter (1.4, $59 \mathrm{~kW}$ MPI engine with MQ 200 gearbox) which formed $0.9 \%$ of the total powertrain's mass, could be observed.
Further work could focus on more detailed analysis of certain issues, impact of engine's liquid media, simplification of the method (its laboriousness) while maintaining the measurement accuracy.

\section{References}

1. F. Vlk, Zkoušení a diagnostika motorových vozidel, Nakladatelství a vydavatelství vlk, BRNO (2001)

2. Z. Bradský, R. Vrzala, Mechanika III. /Dynamika/, VŠST, Liberec (1990)

3. 15. Aachen Colloquium, EUROGRESS Aachen (2006)

4. 5. Tag des Fahrwerks, Institut für Kraftfahrwesen Aachen - RWTH Aachen (09.10.2006)

5. Technická zpráva firmy Škoda Auto At 3374-D, odd. $1-231$

6. Podklady firmy Škoda Auto

7. www.auto.cz

8. M. Brzenina, M. Dvořák, Z. Kalousek, P. Salač, J. Staněk, Matematika 4, TU v Liberci, Liberec (1996)

9. www.wikipedie.cz

10. H. Gortz, Identifikation von Fahrzeugträgheitsparametern in Fahrversuchen und auf Prüfständen, Institut für Kraftfahrwesen Aachen der RWTH Aachen, ISBN 978-3-925194-89-4

11. A. Best, Moment of inertia measurement system, MIMS Description and Specification, Anthony Best Dynamics (2004)

12. R. Sharp, The Measurement of Mass and Inertial Properties of Vehicles and Components, Cranfield University, Cranfield UK (1997)

13. D. Andreatta, G. Heydinger, R. Bixel, D. Coovert, Inertia measurements of large military vehicles, 2001 SAE World Congress Detroit (2001-01-0792)

14. P. Brabec, R. Voženílek, Elipsoid setrvačnosti agregátu - Škoda Auto $1.676 \mathrm{~kW}$ TDI (DPF) Common Rail + MQ250. ZPRÁVA SM595/2008. TU v Liberci, Liberec (2008)

15. Pavel Brabec, Experimentální určení polohy a rozměrů elipsoidu setrvačnosti hnacího agregátu. Teze disertační práce. Technická univerzita v Liberci. (2009) 